\title{
Practical Receipt-Free Sealed-Bid Auction in the Coercive Environment
}

\author{
Jaydeep Howlader ${ }^{1}$, Sanjit Kumar Roy ${ }^{2}$, and Ashis Kumar $\mathrm{Mal}^{3}$ \\ 1 National Institute of Technology, Durgapur, India \\ jaydeep.howlader@it.nitdgp.ac.in \\ 2 sanjit_it@yahoo.co.in \\ 3 ashis.mal@ece.nitdgp.ac.in
}

\begin{abstract}
Sealed-Bid auction is an efficient and rational method to establish the price in open market. However sealed-bid auctions are subject to bid-rigging attack. Receipt-free mechanisms were proposed to prevent bid-rigging. The prior receipt-free mechanisms are based on two assumptions; firstly, existence of untappable channel between bidders and auction authorities. Secondly, mechanisms assume the authorities to be honest (not colluding). Moreover the bandwidth required to communicate the receipt-free bids is huge. This paper presents a sealed-bid auction mechanism to resist bid-rigging. The proposed method does not assume untappable channel nor consider the authorities to be necessarily honest. The proposed mechanism also manages the bandwidth efficiently, and improves the performance of the system.
\end{abstract}

\section{Introduction}

Sealed-bid is a form of auction mechanism where bids are submitted in sealedenvelop. The bids are remained sealed until the schedule time of opening. No bids are accepted after the schedule time of opening. During opening the sealed-bids are opened and the winning price and/or winner(s) are determined. It is rather delicate to implement a sealed-bid auction in the electronic media as there are various essential security requirements to be realized. Moreover, the adversarial behavior of the entities (insider or outsider) may lead to the failure of a naively implemented system. Unlike the outsiders' threat, the adversarial behavior of the insiders are often difficult to counter. For example:

- Auctioneer (insider) opens the bid prior to the schedule opening and conveys the bid-values to the adversary $[14,4]$. Thus fails to meet the confidentiality of bid property.

- Auctioneer allows certain bidder(s) to withdraw or submit unlawfully. Thus fails to meet the fairness property.

- Auctioneer deliberately suppresses some of the valid bids to make a certain bidder to be the winner. Thus fails to meet the correctness property.

- Auctioneer discloses all the bidding prices and the identity of the corresponding bidders after the opening. Thus fails to meet the privacy of the bidder [29] property. 
- Coercer (insider entity) used to corrupt the authorities to retrieve critical information which may yield to bid-rigging [18]. Thus fails to meet the uncoercibility property.

During the last couple of decades sealed-bid auction mechanisms were studied and analyzed in various literatures. In spite of satisfying various security requirements (confidentiality, privacy, fairness, correctness etc.), sealed-bid auction mechanisms are subject to bid-rigging attack. Bid-rigging is a form of coercing where the powerful adversary (e.g. mafia) commands the other bidders to bid as per his choice so that he could win the auction by bidding unreasonably low value. Though the bids are submitted securely, coercer used to enforce the bidders to disclose all the private parameters (e.g. secret randomness, keys etc.) correspond to their secret bids. Thus coercer verifies whether the bidders obey his command. The coercer may corrupt some of the authorities and retrieves vital information that would indulge coercing.

\subsection{Related work}

There have been substantive research works on sealed-bid auction. Franklin \& Reiter [14] first proposed a protocol for secure electronic auction. Kikuchi et.al. [16] proposed the multi-round auction protocol for tie-breaking. Naor et.al. [20] proposed a two-server auction mechanism that protected the privacy of the bidders. In the sequel we include the recent works as $[27,9,6,3]$. However, those mechanisms have no protection to reveal the private inputs if the bidder is willing to do so. In spite of satisfying variety of security requirements, the prior mechanisms are unable to provide bid-rigging.

Abe \& Suzuki [18] first introduced the receipt-free mechanism to counter bidrigging problem. The mechanism was based on threshold encryption [1], with $n$ number of auctioneers. Chen et al. [28] argued that Abe \& Suzuki's mechanism [18] could not provide receipt-freeness to the winning bidder. Moreover, the mechanism failed to provide receipt-freeness in the presence of colluding auctioneer(s). Chen et al. proposed another receipt-free auction mechanism [28]. In their mechanism, seller along with the bidder jointly constructed the receipt-free bid. They argued that, seller would not be colluded due to benefit collision. Her et.al. countered their argument and showed that seller could also be colluded when she tried to make a special bidder to be the winner. Her et al. further proposed another receipt-free auction mechanism [29] based on anonymous channel and pseudo ID. The mechanism required prior bidders' registration. Nevertheless, their mechanism failed to provide receipt-freeness if the registrar was dishonest. Huang et al. [30]proposed some improvement of Abe \& Suzuki's mechanism [18] while reducing the bandwidth of bids, but could not overcome the problem related to dishonest auctioneer(s). Later on Howlader et al. [10] attempted another receipt-free mechanism based on multi-party computation. However the mechanism failed to provide receipt-freeness as the bidders' verification process carried the receipt of the bid. 


\begin{tabular}{|c|c|c|c|c|c|c|}
\hline $\begin{array}{l}\text { Constraints \& As- } \\
\text { sumptions }\end{array}$ & \begin{tabular}{lr|} 
Aber $\quad \&$ \\
Suzuki $[18]$ \\
\end{tabular} & $\begin{array}{l}\text { Chen } \\
\text { et al. }[28]\end{array}$ & \begin{tabular}{|l|l} 
Her \\
et al. $[29]$
\end{tabular} & $\begin{array}{l}\text { Huang } \\
\text { et al. [30] }\end{array}$ & $\begin{array}{l}\text { Howlader } \\
\text { et al }[10]\end{array}$ & $\begin{array}{l}\text { Gao } \\
\text { et al. }[8]\end{array}$ \\
\hline Untappable channel & one way & both way & one way & one way & one way & $\begin{array}{ll}\text { not } & \text { speci } \\
\text { fied } & \\
\end{array}$ \\
\hline Ano & $x$ & $x$ & $\sqrt{ }$ & $x$ & & $\bar{x}$ \\
\hline Hone & $\begin{array}{l}\text { All honest } \\
\text { auctioneer }\end{array}$ & $\begin{array}{l}\text { honest } \\
\text { seller }\end{array}$ & $\begin{array}{ll}\text { honest reg- } \\
\text { istrar }\end{array}$ & $\begin{array}{l}\text { all honest } \\
\text { auctioneer }\end{array}$ & \begin{tabular}{|l|} 
at least one \\
honest sealer
\end{tabular} & $\begin{array}{l}\text { honest auc- } \\
\text { tioneer }\end{array}$ \\
\hline Band & $O(l \times n)$ & $O(l)$ & $O(c)$ & $O(\log l \times n)$ & $O(l)$ & $O(c)$ \\
\hline
\end{tabular}

Table 1: The physical constrains and assumptions made in various sealed-bid auction mechanisms. $l$ denotes the length of the price list, $n$ denotes the number of auctioneers and $c$ denotes constant

Some impractical assumptions: The above mechanisms are based on the two assumptions:

Firstly, the availability of untappable channel ${ }^{4}$ between bidders and authorities (auctioneers, seller, sealer etc.). However, untappable channel is often impractical and difficult to deploy. However, some techniques based on deniable encryption $[21,12]$ ware proposed in $[24,13]$ to relax the untappable channel. The notion of deniability allows the bidders to plausibly evade the coercer. However, those techniques fail in the presence of colluding authorities [11]. Later on Howleder et. al. introduced 'Coercing Resistant Mix (CRM)' [11] which integrated deniability with anonymity to transients the physical requirement of untappable channel.

Secondly, the prior receipt-free mechanisms consider the authorities to be honest. More specifically, the authorities not only execute the protocol honestly, but also avoid any such conspiracy that may leak certain information to the coercer.

\subsection{Our Contribution}

We withdraw the untappable channel, henceforth coercer can intercept the public transcripts at any extend. Furthermore, we consider a broader notion of coerciveness rather than only receipt-freeness. Coercer may collude some of the authorities who execute the protocol correctly but reveal certain information to the coercer in order to indulge coercing. We replace the untappable channel with CRM [11]. CRM allows the adversary to intercept the public transcripts, but provides the bidders to formulate 'fake bids' such that, adversary could not able to distinguish between the fakes and the trues. On the other hand, untraceable delivery of messages restricts the recipient (authority) to link 'who-bids-what'. Though the recipient recives decrypted messages, but unable to determine 'whobids-what'.

Based on the cryptographic techniques, the receipt-free auction mechanisms are categorized in two classes. The mechanisms $[18,30]$ are based on threshold secret sharing which outputs committed transcripts. However, those mechanisms fail to provide receipt-freeness if any one of the authority reveals his share. Whereas the mechanisms $[28,29]$ are based on designated-verifiability of

\footnotetext{
${ }^{4}$ A channel that provides perfect security in an information-theoretic sense. Even
} encryption does not provide an untappable channel 

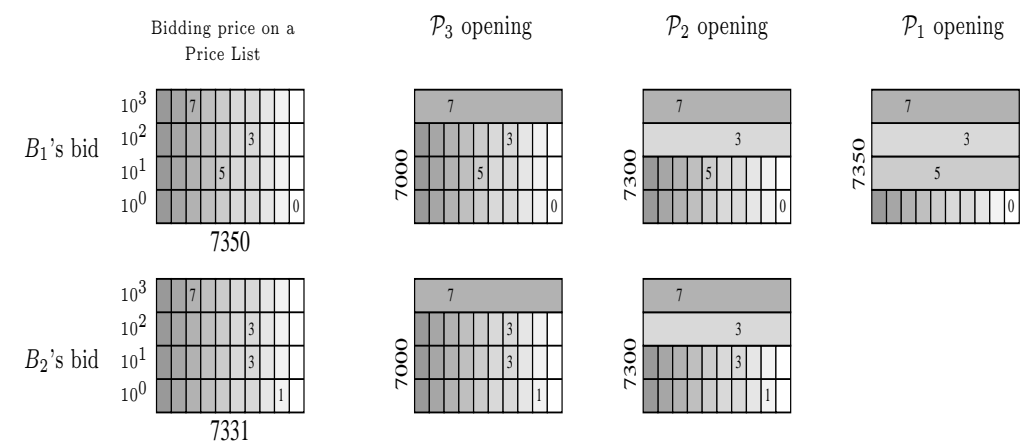

Fig. 1: Opening of two bid-vectors $B_{1}: 7350$ and $B_{2}: 7331$. During the opening of $\mathcal{P}_{1}$ the bid-vector $B_{1}$ is extracted while $B_{2}$ is excluded as $P_{1,5}$ appears before $P_{1,3}$.

re-encryption proof [2], where bidder and authority (either seller, auctioneer) collaboratively form the receipt-free bids. Nevertheless, those schemes also fail if the entities are not trustworthy.

The proposed mechanism is based on secure multi-party computation [5, 25]. The sealing operation is done with respect to a private key which is distributed among a set of qualifying sealers. A quorum of qualifying sealers performs the sealing operation form the sealed-bids. Unlike the prior mechanisms the proposed scheme guarantees receipt-freeness even at least one of the authority remain honest (not colluded).

\section{Preliminaries}

Three main building blocks are used in the proposed receipt-free mechanism. They are Deniable Encryption, Coercer Resistant MIX and Distributed Key Generation.

A Plan-Ahead Deniable Encryption (PDE) [21,12] outputs the cipher $c_{d}$ such that, the encryption of the fake and the true messages look alike. The PDE consists of three algorithms $P D E(E n c, D e c, \varphi)$. The encryption $(E n c)$ is defined as $E n c^{-}\left(m_{t}, p k, r_{t}\right)$, where $m_{t}$ is the true message, $p k$ is the public key and $r_{t}$ is the true randomness, and outputs a cipher $c$. However, Enc produces deniable cipher $c_{d}$ when executed with another parameter called fake message $m_{f}$, as $E n c^{m_{f}}\left(m_{t}, p k, r_{t}\right)$. PDE allows the sender to evade coercion by producing $m_{f}$ instade of $m_{t}$. The decryption (Dec) is defined as $\operatorname{Dec}\left(c\right.$ or $\left.c_{d}, s k\right)$, where $s k$ is the private key and outputs the plaintext $m_{t}$ with negligible decryption error. The faking algorithm $(\varphi)$ is defined as $\varphi\left(c_{d}, m_{t}, m_{f}\right)$ and outputs the fake randomness $r_{f}$ such that $E n c^{-}\left(m_{t}, p k, r_{t}\right)$ and $E n c^{-}\left(m_{f}, p k, r_{f}\right)$ look alike.

MIX (MIX-cascade) is a system consists of a finite number of nodes and provides anonymous communication $[17,19,15]$. MIX takes a list of ciphertexts as input and outputs a random permutation of the plaintexts. Every node performs a cryptographic transformation and a random permutation, and forwards the list 
to the next node. We denote MIX operation as $\operatorname{MIX}\left(E n c_{p k}\left[m_{1}, \ldots, m_{N}\right]\right) \rightarrow$ $\prod\left[m_{1}, \ldots, m_{N}\right]$ where $p k$ is the public key of the MIX and $\prod$ denotes a random permutation of the list. Unlike the general MIXes those take non-probabilistic ciphers $[25,26]^{5}$ as input, CRM takes deniable ciphers as input. Deniability allows the sender to plausibly deny the true message while anonymity restricts the dishonest recipients to retrace the senders of individual messages.

Distributed Key Generation (DKG) allows a set of $n$ entities to generate jointly a pair of public-private key according to the distribution defined by the underlying cryptosystem. The public key is output in the clear, the private key is secretly shared among the $n$ entities via a threshold encryption scheme. A robust and efficient DKG protocol is proposed by Gennaro et.al. [23] to share the secret $x$ amongst a set of qualifying entities and the makes $y=g^{x}$ public. The protocol is able to identify the malicious entities and computes the publicprivate values with the inputs of the qualifying entities. DKG is denoted as $D K G_{P}\left(s_{1}, \ldots, s_{n}\right) \rightarrow(h, x, \mathcal{P})$, where $P$ is the set of $n$ entities, $s_{i}$ is the random secret initiated by the entity $P_{i} \in P, h=g^{x}$ is the public value, $x=f_{s_{i} \in \mathcal{P}}\left(s_{i}\right)$ is the secret shared amongst the entities $P_{i} \in \mathcal{P}$ and $\mathcal{P}$ is the set of qualifying entities.

\section{Receipt-free Sealed-Bid Auction}

The receipt-freeness is proposed to prevent bid-rigging in sealed-bid auction. Following are the entities of the proposed receipt-free auction:

\subsection{Entities}

- There is a finite set of bidders denoted as $B=\left\{B_{1}, B_{2}, \ldots, B_{m}\right\}$.

- There is a finite set of sealers denoted as $S=\left\{S_{1}, S_{2}, \ldots, S_{k}\right\}$. Sealer is an authority who executes sealing operation and forms the receipt-free bid.

- There is a single auctioneer. The auctioneer is responsible to open the bids (with the cooperation of sealers) and determines the winning price and winner.

- Coercer is an adversary who indulges bid-rigging. Coercer is able to impel the bidders to reveal all their private data (keys and randomnesses). Furthermore the coercer is allowed to intercept the public transcripts and also corrupts some of the sealers to retrieve critical information that may yield coercing.

- We use a Bulletin Board $(\mathcal{B B})$. This is a publicly accessible memory with read and appendive-write access.

- We integrate CRM in place of untappable channel

\footnotetext{
${ }^{5}$ probabilistic encryption uses randomness in encryption so that, when encrypting the
} same message several times it will, in general yield different ciphertexts 


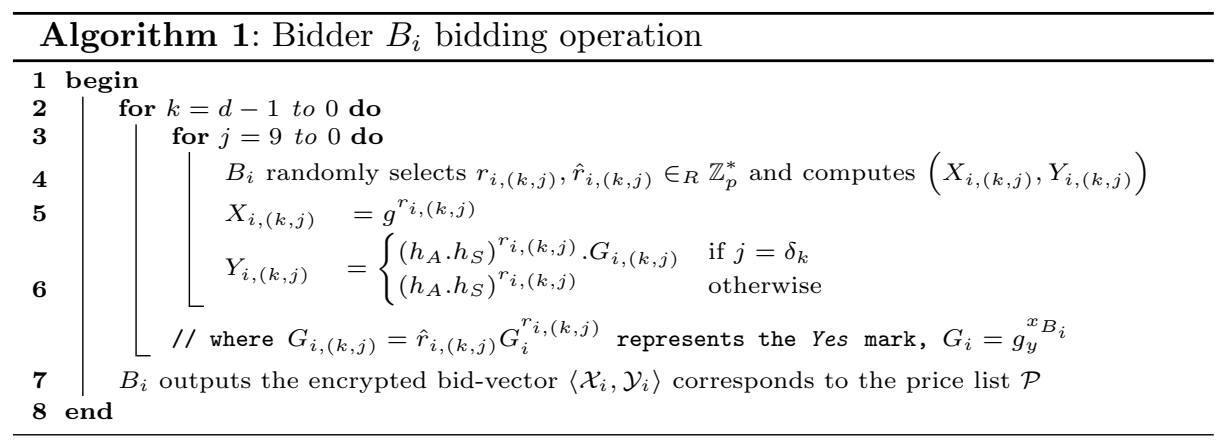

\subsection{System Setting}

Let $p, q$ be large primes such that $q$ divides $p-1, G_{q}$ be the unique subgroup of $\mathbb{Z}_{p}^{*}$ of order $q$, and $g \in \mathbb{Z}_{p}^{*}$ is an element of order $q$. Following we define the keys of different entities. The operations are closure to the multiplicative group $\mathbb{Z}_{p}^{*}$.

- Bidder $B_{i}$ 's private key be $x_{B_{i}} \in \mathbb{Z}_{p}^{*}$ and public key be $h_{B_{i}}=g^{x_{B_{i}}}$.

- Auctioneer's private key be $x_{A} \in \mathbb{Z}_{p}^{*}$ and public key be $h_{A}=g^{x_{A}}$.

- The sealers execute the Distributed Key Generation (DKG) protocol [23,22] that outputs a set of qualifying sealers denoted as $Q U A L$ (of $k$ sealers) with the public key $h_{S}$. Each member $S_{i} \in Q U A L$ has his private key as $x_{i}$ such that any quorum of $t>k / 2$ sealers denoted as $Q R M \subseteq Q U A L$ are able to seal the bidders' encrypted bid-vectors. Without loss of generality, we assume that $Q R M=\left\{S_{1}, S_{2}, \ldots, S_{t}\right\}$. Sealer $S_{i} \in Q R M$ configures his sealing key as $x_{S_{i}}=f_{i}(0)$ where $f_{i}(x)=\lambda_{i j} x_{i}$ is a polynomial of degree $t . \lambda_{i j}{ }^{6}$ is the Lagrange interpolation coefficient for the sealer $S_{i}$.

- After configuring the $Q R M$ each sealer $S_{i} \in Q R M$ publishes his public key for sealing as $h_{S_{i}}=g^{x_{S_{i}}}$. We denote $h_{S / S_{1}, S_{2}, \ldots, S_{r}}=h_{S}\left(h_{S_{1}} h_{S_{2}} \ldots h_{S_{r}}\right)^{-1}$. Intuitively $h_{S / S_{1}, \ldots, S_{t}}=1$

- $g_{y} \in \mathbb{Z}_{p}^{*}$ be an element of order $q$ indicates the YES Mark.

- Let the maximum estimated price of the item is lesser the $10^{d}$. Auctioneer publishes the price list $\mathcal{P}$ consisting of $d$ ordered vectors. We denote $\mathcal{P}:=$ $\mathcal{P}_{d-1}, \mathcal{P}_{d-2}, \ldots, \mathcal{P}_{0}$ where every $\mathcal{P}_{i}$ consists of 10 elements and denoted as $\mathcal{P}_{i}:=$ $P_{i 9}, P_{i 8}, \ldots, P_{i 0}$. The element $P_{i j}$ represents the value $j \times 10^{i}$. Thus the decimal value of $\delta_{d-1} \delta_{d-2} \ldots \delta_{0}$ has an equivalent representation as $\sum_{i=0}^{d-1} P_{i \delta_{i}}$. Fig 1 describes the bid-vector representation of the decimal value (bid value).

\section{Receipt-free sealed-bid auction mechanism}

The receipt-free sealed-bid auction mechanism is consisting of four phases: bidding, sealing,opening and trading.

\footnotetext{
${ }^{6}$ Lagrange interpolation coefficient for the $i^{t h}$ sealer is $\lambda_{i j}=\prod_{\substack{i \neq j \\ 1 \leq j \leq t}} \frac{x-j}{i-j}$
} 


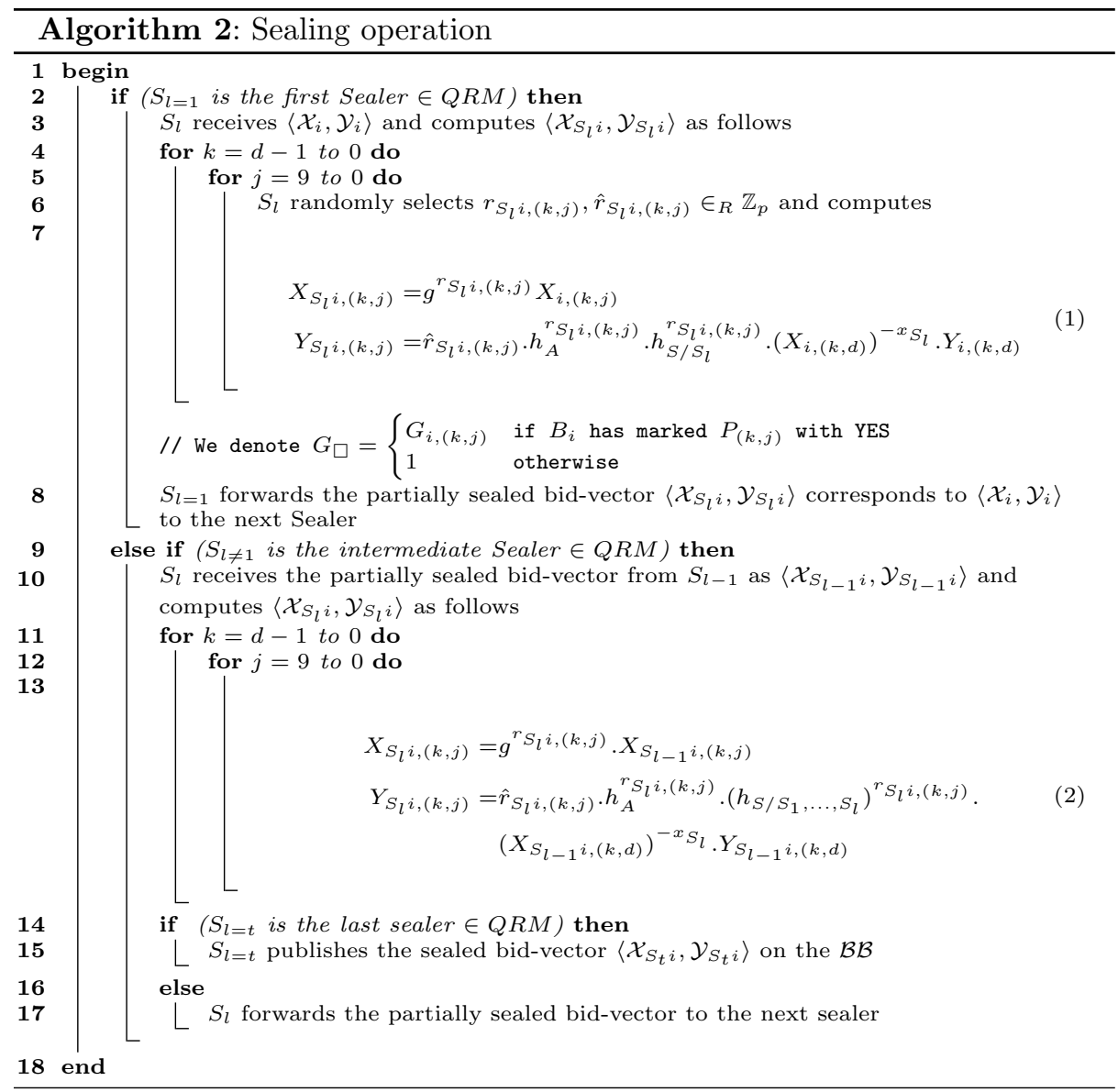

Bidding: Every bidder $B_{i} \in B$ determines his bidding price and constructs the encrypted bid-vector as follows:

- Let $\delta_{d-1} \delta_{d-2} \ldots \delta_{0}\left(0 \leq \delta_{i} \leq 9\right)$ be the decimal representation of the bidding price. $B_{i}$ executes Algorithm 1 to output the encrypted bid-vector $\left\langle\mathcal{X}_{i}, \mathcal{Y}_{i}\right\rangle$.

- $B_{i}$ marks the price indices $P_{k \delta_{k}}(0 \leq k \leq d-1)$ with $Y e s$ while encrypting the price list $\mathcal{P}$.

$B_{i}$ constructs a fake encrypted bid-vector as $\left\langle\overline{\mathcal{X}}_{i}, \overline{\mathcal{Y}}_{i}\right\rangle$ and forwards the deniable cipher Enc ${ }^{\left\langle\overline{\mathcal{X}}_{i}, \overline{\mathcal{Y}}_{i}\right\rangle}\left(\left\langle\mathcal{X}_{i}, \mathcal{Y}_{i}\right\rangle, p k_{C R M}, r_{t}\right)$ to the CRM. CRM accumulates a batch of deniable ciphers and anonymously delivers the batch to the $Q U A L$.

Sealing: A quorum of sealers (denoted as $Q R M \subset Q U A L$ ), possessing the public key as $h_{S}$, performs the sealing operation. The encrypted bid-vectors are processed by at least $t>k / 2$ sealers from the $Q R M$. Every sealer $S_{l} \in Q R M$ executes the Algorithm 2 and outputs the partially sealed bid-vector. During sealing, every sealer $S_{l}$ engraves his secret randomness, $r_{S_{l} i,(k, j)} \& \hat{r}_{S_{l} i,(k, j)}$ and 
nullifies his key component, $h_{S_{l}}$ (in Algorithm 2, equation $1 \& 2$ ) from the partially sealed bid-vector. After $t$ sealing operation sealer $S_{t}$ publishes the sealedbid on the $\mathcal{B B}$.

Bid Verification (BV): The inherent property of receipt-freeness is the inability to prove to any one how a bidder has bid. However, receipt-freeness allows the bidder to verify the correctness of the sealing operation. Algorithm 3 describes the BV mechanism. The BV does not reveal the secret value i.e, even the coercer observes the process of BV, bidder can execute the BV correctly without revealing any partial information related to his secret. BV is done with respect to the cumulative response $\mathcal{R}_{S_{\mathbf{t}} i k}$ computed by every sealers.

Opening: At the schedule Opening, bids are opened. Bids are opened in decreasing order (starting from the highest price). We define two subprocesses: Evaluating Yes Mark (EYM) for the price vector $\mathcal{P}_{k}$ and Extracting Bids having Yes Mark (EBY) on price index $P_{k, j}$.

- EYM: Auctioneer and sealers jointly execute the process. EYM takes input a price vector $\mathcal{P}_{k}$ and output the highest price index $P_{k, j}$ that contains some Yes Marks. Algorithm 4 describes the process.

- EBY: After EYM outputs an index $P_{k, j}$, EBY extracts and outputs a list of bids (sealed-bids) that contains the sealed-bids having Yes Marks on the $P_{k, j}$ index.

The opening phase is initiated with the construction of the list $L$ containing all the sealed bids followed by invoking the subporcess $E Y M\left(L, \mathcal{P}_{d-1}\right) . E Y M$ will output the price index $P_{d-1, w_{d-1}}$ and the list $L_{d-1}$ containing those sealed bids which possess Yes Mark on the index $P_{d-1, w_{d-1}}$. Auctioneer sets the winning price as $w=w_{d-1} x x x$. Auctioneer subsequently iterates the subprocess $E Y M\left(L_{k}, \mathcal{P}_{k}\right)$ (for $k=d-2, \ldots 0$ ) and finally the winning price $w=w_{d-1} \ldots w_{0}$ and the list of winning bids $L_{0}$ is determined.

Trading: The auction mechanism determines the winning bids, but not the winner. The winning bidder claims his winning and executes a zero-knowledge (ZK) protocol with the auctioneer to substantiate his winning. Let $B_{i}$ be the winning bidder and $w=w_{d-1} \ldots w_{0}$ be the winning price. Bidder $B_{i}$ proves $G_{i}$ and $h_{B_{i}}$ have common exponent over $g_{y}$ and $g$ respectively.

For $k=d-1, \ldots 0, B_{i}$ discloses all $\hat{r}_{i,\left(k, w_{k}\right)}$ and proves that $X_{i,\left(k, w_{k}\right)}$ and $G_{i,\left(k . w_{k}\right)} \cdot\left(\hat{r}_{i,\left(k, w_{k}\right)}\right)^{-1}$ have common exponents over $g$ and $G_{i}$ respectively.

The details of the ZK protocol is presented in the Appendix.

\section{Security Analysis}

In this section we present the security properties of the proposed scheme:

Receipt-Freeness: If $A$ is an auction protocol and simulated as

$$
\begin{aligned}
A \triangleq & \operatorname{Bid}\left(\forall i B_{i}, b_{i}, r_{B_{i}}\right)\left|\operatorname{Seal}\left(\forall t S_{t}, r_{S_{t}}, \hat{r}_{S_{t}}\right)\right| \operatorname{out}\left(s b_{i}\right) \mid \\
& \operatorname{Rev}\left(B_{c}, r_{B_{c}}\right)\left|! \operatorname{Rev}\left(\exists h S_{h}, r_{S_{h}}, \hat{r}_{B_{h}}\right)\right| \operatorname{Rev}\left(\forall t S_{t}, r_{S_{t}}, \hat{r}_{S_{t}}\right)
\end{aligned}
$$




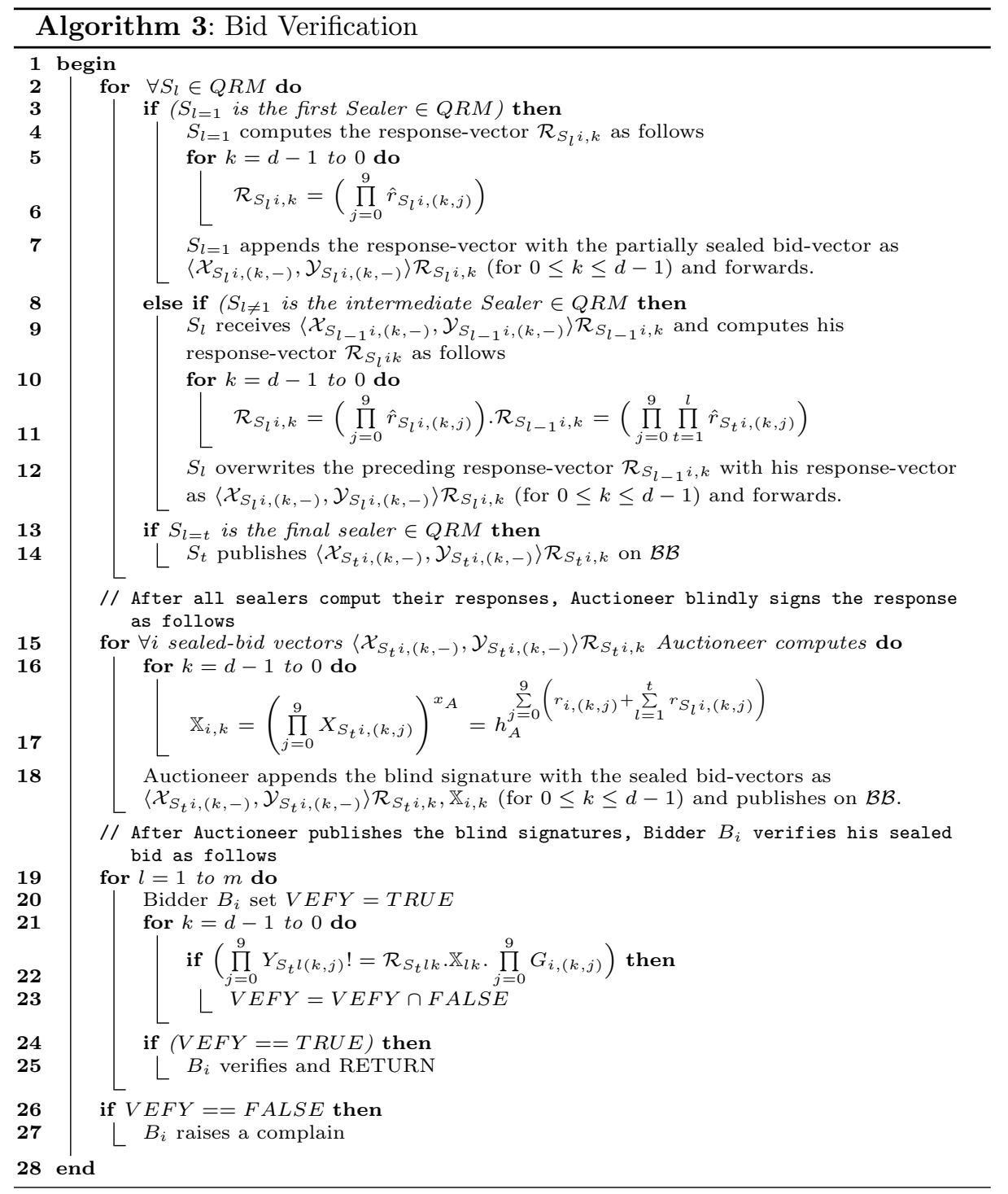




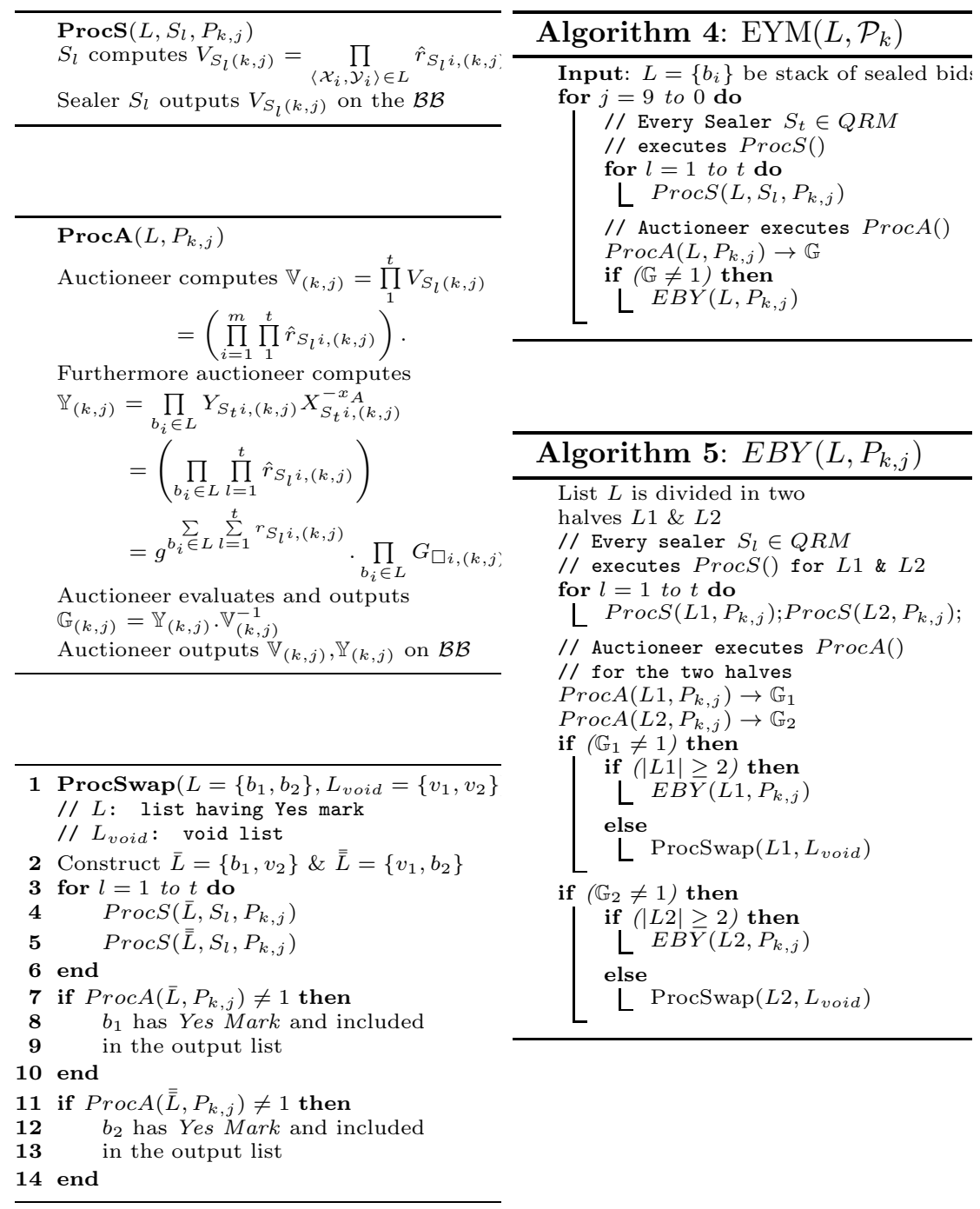


where every bidder $B_{i}$ encrypts his bid $b_{i}$ with the randomness $r_{B_{i}}$, every sealer $S_{t}$ seals the bids with randomness $r_{S_{i}}, \hat{r}_{S_{i}}$ and produces the sealed bids $s b_{i}$, thereafter, the coerced bidder $B_{c}$ and all sealers except the honest sealer $S_{h}$ reveal their secrets. The protocol still conceals the private values. We show that, adversary who may compute buy could not resolve the secret as the private values are

$$
Y_{S_{t} c,(k, j)} \cdot\left(\prod_{l=1 l \neq h}^{t} \hat{r}_{S_{l} c,(k, j)} \cdot h_{A}^{\left(r_{c,(k, j)}+{ }_{l=1 l \neq h}^{t}{ }^{r_{S_{l}} c,(k, j)}\right)}\right)^{-1}=\hat{r}_{S_{h} c,(k, j)} \cdot G_{\square}
$$

blinded with the honest bidder's randomness $\left(\hat{r}_{S_{h} c(k, j)}\right)$. Similarly, bidder $B_{c}$ could flip the Yes-to-No and vice-versa. The proposed mechanism ensures receiptfreeness as adversary could not distinguish between a situation where $B_{c}$ reveals his true secret and the situation where he produces fake secret.

Correctness: The auction mechanism declares the winning price and keeps all the loosing bids secret. The correctness defines the ability to verify the outcome of the auction by any entity. Let auctioneer declared $w=w_{d-1} w_{d-2} \ldots w_{0}$ as the winning price. Therefore during opening the subprocesses $\operatorname{ProcS}()$ and $\operatorname{ProcA}()$ have published all $V_{S_{i}(k, j)}$ and $\mathbb{V}_{(k, j)} \& \mathbb{Y}_{(k, j)}\left(0 \leq k \leq d-1,9 \geq j \geq w_{k}\right)$ on $\mathcal{B B}$ on the $\mathcal{B B}$. Any one who wants to verify the correctness of the auction result can examine the result with the information published on the mathcalBB.

Nonrepudiation: We assume that bidder bids honestly. The opening of bids only determines the winning price and the list of winning bids, but winner is not determined. Bidder executes the ZK protocol to substantiate his winning. However, the odd may happen, when the winning bidder does not respond. We present the mechanism to identify the winning bidder while he has not responded. Let $w=w_{d-1} w_{d-2} \ldots w_{0}$ be the winning price and $L_{0}$ be the list of sealed-bids extracted as the winning bid(s). Let $\left\langle\mathcal{X}_{S_{t}}, \mathcal{Y}_{S_{t} i}\right\rangle \in L_{0}$ be an winning bid. In the opening phase, procedure ProcSwap() computes the Yes Mark on every $P_{k, w_{k}}$ of the winning bid. Let $\mathcal{G}=\left\{G_{i\left(k, w_{k}\right)} \mid 0 \leq k \leq d-1\right\}$ be the set of Yes Marks computed by ProcSwap() during opening. Now auctioneer has to identify the bidder(s) who had bid with the above set of Yes Marks. Auctioneer initiates the following:

- Auctioneer asks all sealer $S_{l} \in Q R M$ to publish the initial encrypted pricevectors on $\mathcal{B B}$. Thus all $\left\langle\mathcal{X}_{i}, \mathcal{Y}_{i}\right\rangle$ (for $i=1,2, \ldots m$ ) appears on the $\mathcal{B B}$.

- Auctioneer asks the bidders to substantiate their encrypted bids for every $P_{k, w_{k}}$ indices. That is, all the losing bidder $B_{i}$ will show that:

1. he knows the discrete logarithm of $X_{i\left(k, w_{k}\right)}$ (say $\left.r_{i\left(k, w_{k}\right)}\right)$ and

2. shows that $Y_{i\left(k, w_{k}\right)}=\left(h_{S} h_{A}\right)^{r_{i\left(k, w_{k}\right)}}$.

However, the winning bidder $B_{w}$ will fail to establish the second as he had computed $Y_{w\left(k, w_{k}\right)}=\left(h_{S} h_{A}\right)^{r_{w\left(k, w_{k}\right)}} \cdot G_{w\left(k, w_{k}\right)}$.

\section{Performance}

The proposed scheme improves the performance by reducing the bandwidth of the receipt-free bids. The existing receipt-free auction mechanisms e.g. [18, 


\begin{tabular}{|l||l|l|l|}
\hline $\begin{array}{l}\text { No. of } \\
\text { Rounds }\end{array}$ & $\begin{array}{l}\text { Abe \&uzuki [18] } \\
\text { Sul }\end{array}$ & $\begin{array}{l}\text { Huang } \\
\text { et al. }[30]\end{array}$ & $\begin{array}{l}\text { proposed } \\
\text { mechanism }\end{array}$ \\
\hline $\begin{array}{l}\text { During } \\
\text { Bidding }\end{array}$ & $n m L$ & $n m(\log L)$ & $m\left(\log _{10} L\right)$ \\
\hline $\begin{array}{l}\text { During } \\
\text { Opening }\end{array}$ & $t \geq n / 2$ & $t \geq n / 2$ & $\begin{array}{l}\text { worst case } \\
10 d \quad \text { and } \\
d m\end{array}$ \\
\hline
\end{tabular}

\begin{tabular}{|l||l|l|}
\hline $\begin{array}{l}\text { No. of } \\
\text { rounds) }\end{array}$ & $\begin{array}{l}\text { Chen } \\
\text { et al. }[28]\end{array}$ & $\begin{array}{l}\text { Her } \\
\text { et al. }[29]\end{array}$ \\
\hline $\begin{array}{l}\text { During } \\
\text { Bidding }\end{array}$ & at least $m n L$ & at least $m n$ \\
\hline $\begin{array}{l}\text { During } \\
\text { Opening }\end{array}$ & at most $L$ & at most $L$ \\
\hline
\end{tabular}

Table 2: Number of message exchanges in various auction mechanisms

\begin{tabular}{|c|c|c|c|c|c|c|c|c|}
\hline & \multicolumn{4}{|c|}{ Howlader et. al. $[10]$} & \multicolumn{4}{|c|}{ proposed mechanism } \\
\hline \multirow{3}{*}{$\begin{array}{ll}\text { key } & \text { size } \\
\text { (bits) }\end{array}$} & \multicolumn{2}{|c|}{ Bidding time in sec. } & \multicolumn{2}{|c|}{ Sealing time in sec. } & \multicolumn{2}{|c|}{ Bidding time in sec. } & \multicolumn{2}{|c|}{ Sealing time in sec. } \\
\hline & \multicolumn{2}{|c|}{ price list length } & \multicolumn{2}{|c|}{ price list length } & \multicolumn{2}{|c|}{ price list length } & \multicolumn{2}{|c|}{ price list length } \\
\hline & 5,000 & 10,000 & 5,000 & 10,000 & 5,000 & 10,000 & 5,000 & 10,000 \\
\hline 512 & 14 & 29 & 37 & 75 & 0.22 & 0.22 & 0.48 & 0.48 \\
\hline 1024 & 98 & 195 & 248 & 495 & 1.48 & 1.48 & 2.76 & 2.76 \\
\hline 1536 & 308 & 615 & 766 & 1515 & 4.63 & 8.45 & 5.69 & 10.24 \\
\hline
\end{tabular}

Table 3: Time latency for Bidding and Sealing operation

$28,10]$ require huge bandwidth to communicate the encrypted bids. Table 1 presents the bandwidth requirement for various auction mechanisms. In this section we analyze the bandwidth requirement, communication overhead and computational complexity of the proposed mechanism. Let $L, n$ and $m$ represent the number of bidding price, number of auctioneer/sealer and number of bidder respectively.

We represent the price list as a $d$-tuples of constant length ordered-vectors. A price list of $d$ vectors is capable to represent the value up to $10^{d}$. Reduction in the size of price list decreases the bandwidth requirement and computational overhead. We estimate bandwidth of every receipt-free bid is $O\left(\log _{10} L\right)$.

Moreover, The proposed mechanism defines less number of message exchange between the entities. Table 2 presents the number of message exchanges required to execute the proofs $\& 3$ verification. We also present the average time latency of bidding and sealing operation with varying key size and number of bidding price. Table 3 shows the comparison of time latency between to mechanisms.

\section{Conclusion}

The proposed auction scheme attempts to solve two existing problems; firstly, it provides receipt-freeness without any untappable channel and secondly, it ensures uncoerciveness even in the presence of colluding authorities. The mechanism guarantees uncoerciveness even all the sealer except one are dishonest. No prior registration of bidder is required. So any one who possesses the required key may participate in the auction. Bidders are not necessarily be present during opening i.e. ensures 'bid-and-go' concept. The proposed mechanism improves the performance and efficiency by reducing the bandwidth and communication round. 


\section{References}

1. Shamir A. How to share a secret. Commun. ACM, 22(11):612-613, 1979.

2. Lee B. and Kim K. Receipt-free electronic voting scheme with a tamper-resistant randomizer. In ICISC'02, LNCS2587, pages 389-406. Springer-Verlag, 2002.

3. Mihály Bárász, Péter Ligeti, László Mérai, and Daniel A. Nagy. Anonymous sealed bid auction protocol based on a variant of the dining cryptographers' protocol. Periodica Mathematica Hungarica, 65(2):167-176, 2012.

4. Boyd C. and Mao W. Security Issues for Electronic Auctions. HP Laboratories technical report. Hewlett-Packard Laboratories, 2000.

5. Yao Andrew C. Protocols for secure computations. In 23rd Annual Symposium on Foundations of Computer Science, SFCS '82, pages 160-164. IEEE Computer Society, 1982.

6. Wu C-C., Chang C-C., and Lin I-C. New sealed-bid electronic auction with fairness, security and efficiency. J. Comput. Sci. Technol., 23(2):253-264, 2008.

7. Chaum D. and Pedersen T. P. Wallet database with observers. In Advances in Cryptology, CRYPTO 92, LNCS 740, pages 89-105, 1993.

8. Chongzhi Gao, Zheng an Yao, Dongqing Xie, and Baodian Wei. Electronic sealedbid auction with incoercinility. In Electronic Power Systems and Computers, LNEE 99, pages 47-54. Springer-Verlag, 2011.

9. Xiong H., Qin Z., Zhang F., Yang Y., and Zhao Y. A sealed-bid electronic auction protocol based on ring signature. In ICCCAS, pages 480-483. IEEE, 2007.

10. Howlader J., Ghosh A., and Pal T. D. Secure receipt-free sealed-bid electronic auction. In IC3, CCIS 40, pages 228-239. Springer, 2009.

11. Howlader J., Kar J., and Mal A. K. Coercion resistant mix for electronic auction. In ICISS, LNCS 7671, pages 238-248. Springer, 2012.

12. Howlader J. and Basu S. Sender-side public key deniable encryption scheme. In ARTCom, pages 9-13. IEEE Computer Society, 2009.

13. Howlader J., Nair V., Basu S., and Mal A. K. Uncoercibility in e-voting and eauctioning mechanisms using deniable encryption. IJNSA, 3(2):97-109, 2011.

14. Franklin M. K. and Reiter M. K. The design and implementation of a secure auction service. IEEE Transactions on Software Engineering, 22(5):302-312, 1996.

15. Sako K. and Kilian J. Receipt-free mix-type voting scheme: a practical solution to the implementation of a voting booth. In EUROCRYPT, pages 393-403, 1995.

16. Hiroaki Kikuchi, Michael Hakavy, and Doug Tygar. Multi-round anonymous auction protocols. Institute of Electronics, Information, and Communication Engineers Transactions on Information and Systems, E82-D(4):769-777, April 1999.

17. Chaum D. L. Untraceable electronic mail, return addresses, and digital pseudonyms. Commun. ACM, 24(2):84-90, 1981.

18. Abe M. and Suzuki K. Receipt-free sealed-bid auction. In ISC, LNCS 2433, pages 191-199. Springer, 2002.

19. Jakobsson M. A practical mix. In EuroCrypt, pages 448-461, 1998.

20. Moni Noar, Benny Pinkas, and Reubew Sumner. Privacy preserving auction and mechanism design. In ACM Conference on Electronic Commerce, pages 129-139. ACM, 1999.

21. Canetti R., Dwork C., Naor M., and Ostrovsky R. Deniable encryption. In CRYPTO 97, pages 90-104, 1997.

22. Gennaro R., Jarecki S., Krawczyk H., and Rabin T. Secure distributed key generation for discrete-log based cryptosystems. In EUROCRYPT, LNCS 1592, pages 295-310. Springer, 1999. 
23. Gennaro R., Jarecki S., Krawczyk H., and Rabin T. Secure distributed key generation for discrete-log based cryptosystems*. Journal of Cryptology, 20(1):51-83, 2007.

24. Zuzana Rjašková. Electronic voting schemes. Master's thesis, Department of Computer Science Faculty of Mathematics, Physics and Informatics Comenius University, Bratislava, 2002.

25. Goldwasser S. and Micali S. How to play any mental game or a completeness theorem for protocols with honest majority. In 19th annual ACM symposium on Theory of computing, pages 365-377. ACM, 1982.

26. Goldwasser S. and Micali S. Probabilistic encryption. Journal of Computer and Systems Sciences, 28(2):270-299, 1984.

27. Ham W., Kim K., and Imai H. Yet another strong sealed-bid auctions. In SCIS, page 1116, 2003.

28. Chen X., Lee B., and Kim K. Receipt-free electronic auction schemes using homomorphic encryption. In ICISC, LNCS 2971, pages 259-273. Springer, 2003.

29. Her Y-S., Imamoto K., and Sakurai K. Receipt-free sealed-bid auction based on mix-net and pseudo id, 2004.

30. Huang Z., Qiu W., Guan H., and Chen K. Efficient receipt-free electronic auction protocol. In SITIS, pages 1023-1028. IEEE Computer Society, 2007.

\section{Appendix}

\section{Proof of Sealing}

Sealer $S_{l}$ receives the partially sealed bid-vector $\left\langle\mathcal{X}_{S_{l-1} i}, \mathcal{Y}_{S_{l-1} i}\right\rangle$ from the preceding sealer $S_{l-1}$, selects $\hat{r}_{S_{l} i,(k, j)}, r_{S_{l} i,(k, j)} \in \in_{R} \mathbb{Z}_{p}$ randomly, performs the sealing operation and forwards the partially sealed bid-vector to the next sealer $S_{l+1}$. Fig. 2 describes the process. The sealing operation of the $S_{l}$ is as follows:

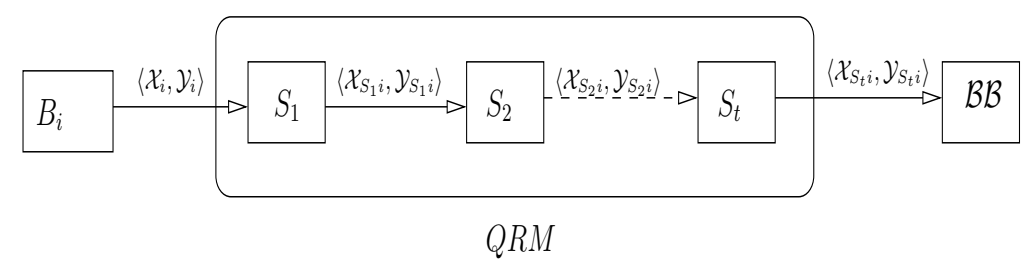

Fig. 2: Sequence of Sealing operation 


$$
\begin{aligned}
& X_{S_{l} i,(k, j)}=g^{r_{S_{l} i,(k, j)}} \cdot X_{S_{l-1} i,(k, j)} \\
& =g^{r} S_{l} i,(k, j) \cdot g^{\left(r_{i,(k, j)}+{ }_{t=1}^{l-1} r_{S_{t} i,(k, j)}\right)} \\
& =g^{\left(r_{i,(k, j)}+\sum_{t=1}^{l} r_{S_{t} i,(k, j)}\right)} \\
& Y_{S_{l} i,(k, j)}=\hat{r}_{S_{l} i,(k, j)} \cdot h_{A}^{r_{S_{l}} i,(k, j)} \cdot\left(h_{S / S_{1}, \ldots S_{l}}\right)^{r_{S_{l}} i,(k, j)} \cdot\left(X_{S_{l-1} i,(k, j)}\right)^{-x} S_{l} \cdot Y_{S_{l-1} i,(k, j)} \\
& =\hat{r}_{S_{l} i,(k, j)} \cdot h_{A}^{{ }^{r} S_{l} i,(k, j)} \cdot\left(h_{S / S_{1}, \ldots S_{l}}\right)^{r_{S_{l}} i,(k, j)} \text {. } \\
& \prod_{t=1}^{l-1} \hat{r}_{S_{t} i,(k, j)} \cdot h_{A}^{\left(r_{i,(k, j)}+{ }_{t=1}^{l-1} r_{S_{t} i,(k, j)}\right)} \cdot\left(h_{S / S_{1} \ldots S_{l}}\right)^{\left(r_{i,(k, j)}+\sum_{t=1}^{l-1} r_{S_{t} i,(k, j)}\right)} \cdot G_{\square} \\
& =\prod_{t=1}^{l} \hat{r}_{S_{t} i,(k, j)} \cdot h_{A}^{\left(r_{i,(k, j)}+\sum_{t=1}^{l} r_{S_{t} i,(k, j)}\right)} \cdot\left(h_{S / S_{1} \ldots S_{l}}\right)^{\left(r_{i,(k, j)}+\sum_{t=1}^{l} r_{S_{t} i,(k, j)}\right)} \cdot G_{\square}
\end{aligned}
$$

After $t$ sealing operation the bid-vector is reduced to

$$
\begin{aligned}
& X_{S_{t} i,(k, j)}=g^{\left(r_{i,(k, j)}+\sum_{l=1}^{t} r_{S_{l} i,(k, j)}\right)} \\
& Y_{S_{t} i,(k, j)}=\prod_{l=1}^{t} \hat{r}_{S_{l} i,(k, j)} \cdot h_{A}^{\left(r_{i,(k, j)}+\sum_{l=1}^{t} r_{S_{l} i,(k, j)}\right)} \cdot\left(h_{\left.S / S_{1} \ldots S_{t}\right)}{ }^{\left(r_{i,(k, j)}+\sum_{l=1}^{t} r_{S_{l} i,(k, j)}\right)} \cdot G_{\square}\right. \\
& =\prod_{l=1}^{t} \hat{r}_{S_{l} i,(k, j)} \cdot h_{A}^{\left(r_{i,(k, j)}+\sum_{l=1}^{t} r_{S_{l} i,(k, j)}\right)} \cdot G_{\square}
\end{aligned}
$$

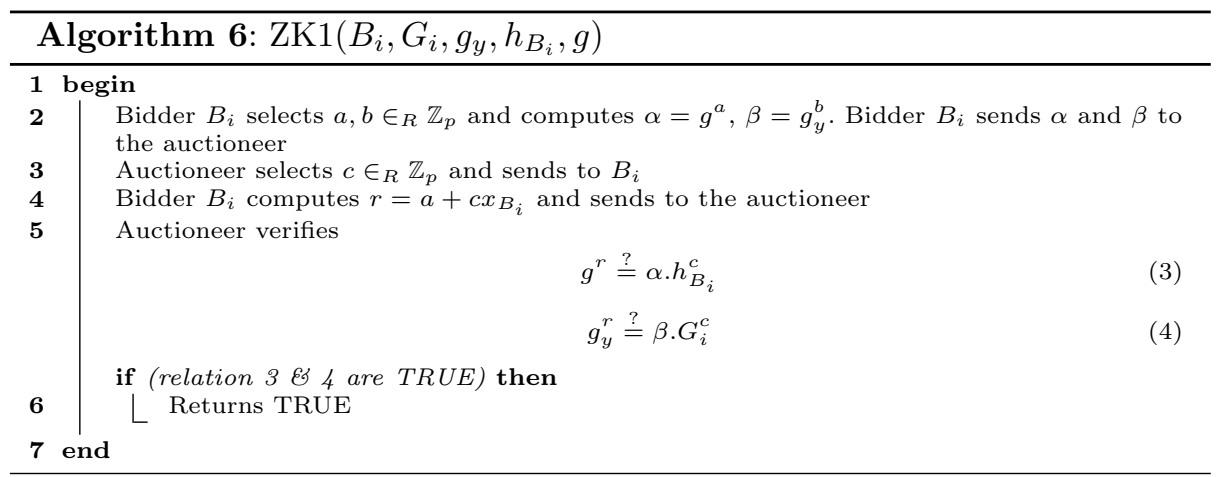

\section{ZK protocol}

Zero-Knowledge (ZK) protocol [7] is a tool by which the prover can prove to another party (the verifier) that a function has been correctly computed, without revealing the secret parameters of the computation. The auction mechanism uses 


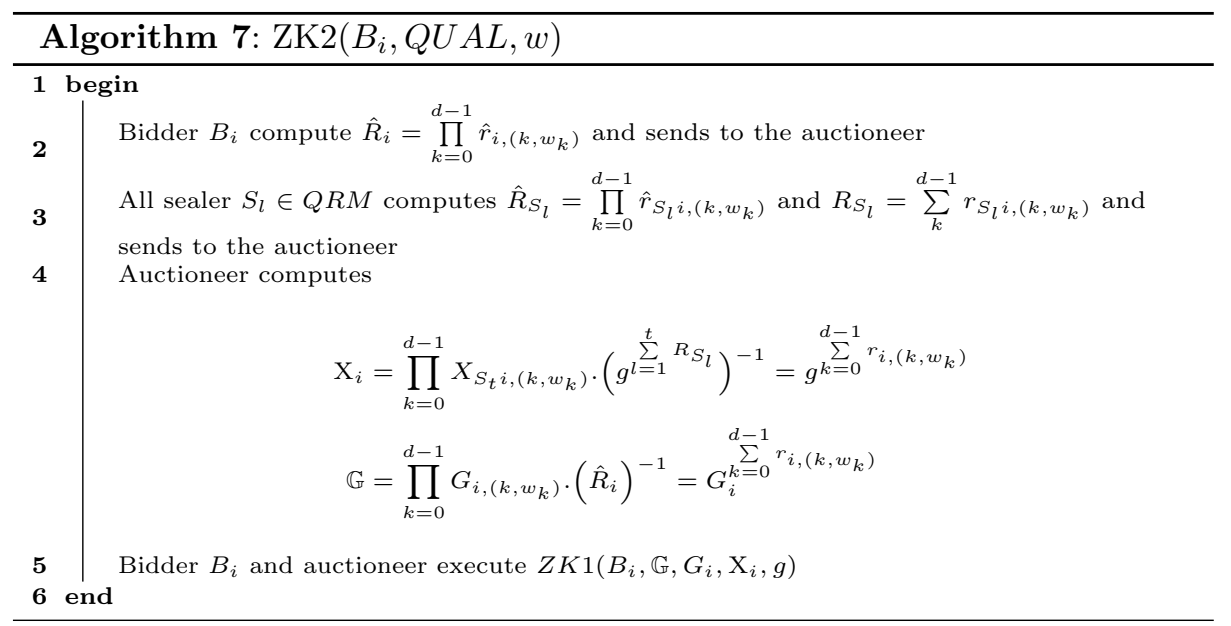

the ZK protocol to determine the winning bidder. Let $w=w_{d-1} \ldots w_{0}$ be the winning price and $B_{i}$ responds as the winner. The bidder $B_{i}$ have to prove the following:

- $B_{i}$ publishes $G_{i}=g_{y}^{x_{B_{i}}}$ and proves that $G_{i}$ and $h_{B_{i}}$ having common exponent $\left(x_{B_{i}}\right)$ over $g_{y}$ and $g$ respectively, without disclosing the secret $x_{B_{i}}$. Algorithm 6 describes the proof.

- For $k=0,1, \ldots d-1, B_{i}$ publishes the product of all $\hat{r}_{i,\left(k, w_{k}\right)}$ and proves that he knows the common exponents over $X_{i,\left(k, w_{k}\right)}$ s and $G_{i,\left(k, w_{k}\right)}$ s. The proof would not be carried on individual items but exercised on the product of all $X_{i,\left(k, w_{k}\right)}$ (for $\left.k=0,1, \ldots d-1\right)$. The Algorithm 7 describes the proof.

\section{Does ProcSwap() vulnerable}

The subprocess $E B Y()$ is a recursive process that partitions the list $L$ into two halves and invokes the ProcSwap(). The Fig 3 shows the process of

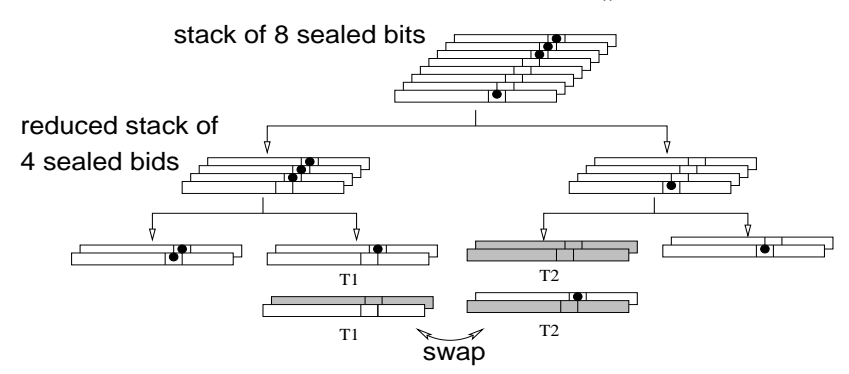

Fig. 3: Process of EBY() partitioning ans swapping operation. $E B Y()$ divides the list into some stacks of sealed bids. Every stack contains only two sealed bids where at least one of them must contains the Yes Mark on the $P_{k, w_{k}}$ index. However, ProcSwap() procedure takes a stack 
(size 2) and demands additional information to determine the bid containing the Yes Mark. We claim that the additional information that is published in order to execute ProcSwap() does not compromise the receipt-freeness property.

Lemma 1. Let $a, b, c \& d \in \mathbb{Z}_{p}$ such that;

$$
\begin{array}{ll}
a . b=k_{1} & c . d=k_{2} \\
a . c=k_{3} & b . d=k_{4}
\end{array}
$$

Though the values of $k_{1}, k_{2}, k_{3} \& k_{4}$ are known, it is computationally infeasible to find the unique solution of $a, b, c \& d$.

Proof. In the above set of equation, any one of the equation is derivable from the other three equations. Let $a . b=k_{1}, c . d=k_{2}$ and $a . c=k_{3}$ are given, the fourth equation can be derivable from the given three equations, that is, $b . d=(a . b) .(c . d) \cdot(a . c)^{-1}=k_{1} \cdot k_{2} \cdot k_{3}^{-1}$. Therefore the above system is effectively consists of three equations with four unknown variables. Henceforth infeasible to determine the unique solution of the $a, b, c \& b$. If $p$ is sufficiently big any random search is inefficient to get the solution of $a, b, c, \& d \square$.

Let $T 1$ be a stack containing two bids $B_{1}$ and $B_{2}$. Also let $T 2$ be another stack containing two void bids $V_{1}$ and $V_{2}$. Therefore the $\mathcal{B B}$ already contains the values

$$
\begin{gathered}
k_{1}=\hat{r}_{S_{1} B_{1},(k, j)} \cdot \hat{r}_{S_{1} B_{2},(k, j)} \\
k_{2}=\hat{r}_{S_{1} V_{1},(k, j)} \cdot \hat{r}_{S_{1} V_{2},(k, j)}
\end{gathered}
$$

(The procedure $\operatorname{ProcS}\left(T 1, S_{1}, P_{k, j}\right)$ and $\operatorname{ProcS}\left(T 2, S_{1}, P_{k, j}\right)$ publish the values) The call to the procedure ProcSwap(T1,T2) demands

$$
\begin{gathered}
k_{3}=\hat{r}_{S_{1} B_{1},(k, j)} \cdot \hat{r}_{S_{1} V_{2},(k, j)} \\
k_{4}=\hat{r}_{S_{2} V_{1},(k, j)} \cdot \hat{r}_{S_{1} V_{2},(k, j)}
\end{gathered}
$$

Knowing the values $k_{1}, k_{2}, k_{3} \& k_{4}$ adversary would not able to resolve the secrets $\hat{r}_{S_{1} B_{1},(k, j)}$ and $\hat{r}_{S_{1} B_{2},(k, j)}$ without better than any random guess. 